PART IV

COMPARISON OF THEORY AND OBSERVATIONS 
A. GRAVITATIONAL EFFECTS 


\title{
72. INTERPRETATION OF LARGE-SCALE SPIRAL STRUCTURE
}

\author{
C. C. LIN \\ Massachusetts Institute of Technology, Cambridge, Mass., U.S.A.
}

\begin{abstract}
The present paper consists of three parts: (1) A general explanation, from a semi-empirical point of view, of the density wave theory and its ramifications, with only a few remarks on those detailed features which have not been stressed before; (2) a statement of our conclusions about the Milky Way System; and (3) a discussion of the problem of the origin of density waves of spiral form.
\end{abstract}

\section{Density Wave Theory of Galactic Spirals: A Semi-Empirical Approach}

\section{A. EXISTENCE OF 'GRAND DESIGN'}

During this conference, several speakers have pointed out that the spiral features in a galaxy are complicated, but there is also no doubt about the existence of a 'grand design', usually two-armed in the inner parts, but often multiple-armed in the outer parts. As properly stressed by Oort (1962) at the Princeton conference, the explanation of the existence of the grand design needed urgent consideration. At the present stage of the development of the theory, now that this grand design is reasonably well understood, it is perhaps more appropriate, if one looks into the future, to pay more attention to the more complicated spiral features on a smaller scale, for here we may learn more about the physical processes in the galaxy. Indeed, Oort has placed slightly greater emphasis on these features in his introductory lecture at this symposium (see p. 1). One might indeed paraphrase his present statement in terms of his 1961 words as follows: Although the general form of the large-scale phenomenon can be recognized in many nebulae, this structure is often hopelessly irregular and broken up.

For the past few years, Shu, Yuan and I myself have been trying to ascertain the grand design of our galaxy by calculating a spiral pattern from the density wave theory (Lin and Shu, 1964, 1966, 1967), in the spirit of the late B. Lindblad, and comparing the theoretical deductions with observational data (Lin et al., 1969; Yuan, 1969). We began our studies by adopting a semi-empirical approach. We followed Oort in dividing the problem of spiral structure into two parts; (a) the persistence of the spiral structure in the presence of differential rotation (or the question of material arms versus density waves), and (b) the origin of this structure. This turns out to be a very fortunate decision, for problem (b) turns out to be a much more difficult theoretical problem than problem (a). Indeed, it is still largely unsolved (cf. Section 3). We shall therefore continue to stress this semi-empirical approach in the present paper.

\section{B. GENERAL FEATURES TO BE EXPLAINED}

I list below ten general observational features which one must consider in dealing with spiral features in galaxies. Their explanation in terms of the density wave theory will be considered in the next section. 
(1) Existence of grand design

The spiral structure extends as a 'pattern' over the 'whole' galactic disk.

(2) Persistence

(a) The spacing between spiral arms, which is used to classify normal spirals into $\mathrm{Sa}, \mathrm{Sb}, \mathrm{Sc}$ types, is correlated with other physical characteristics of the galaxy.

(b) In particular, a smaller nucleus is associated with wider spacing.

(3) Two-armed, trailing

The spiral pattern is in general two-armed and, as far as is known, always trailing.

(4) Multiple-armed structure

Multiple-armed structure is often observed in the outer regions of many galaxies.

(5) Ring structure possible

A ring structure is often observed in the inner part of many galaxies. This structure is very clearly seen in NGC 5364.

(6) Slender strings of beads

The newly formed stars and young $\mathrm{H}$ II regions are arranged neatly like a slender string of beads. This indicates star formation in restricted regions, but simultaneously over a wide front. (It is perhaps this feature that led people to think of magnetic containment as a way to pinch the gas along narrow tubes.)

(7) Dust lanes

The principal dust lanes lie on the inside edge of the bright optical arm, although there are other features associated with dust.

(8) Abundance distribution of $\mathrm{H} \mathrm{II}$

Both the continuum survey of Westerhout and the recombination line observations of Burke, Mezger, and their collaborators Reifenstein and Wilson indicate a marked deficiency of $\mathrm{H}$ II region inside of the ' $3 \mathrm{kpc}$ circle'. This may be compared with the absence of $\mathrm{H}$ II regions within the ring of NGC 5364.

(9) Abundance distribution of $\mathrm{H}_{\mathrm{I}}$

M. Roberts has shown that the peak distribution of $\mathrm{HI}$ extends beyond the bright spiral structure in M 33 and other galaxies. This agrees in general trend with that known in our galaxy: the peak $\mathrm{H}_{\mathrm{I}}$ distribution lies outside of the Hil peak (Westerhout).

(10) Magnetic field

The magnetic field is relatively weak and, in the case of the Orion arm, it lies roughly along the arm (Hiltner).

\section{QSSS HYPOTHESIS}

It is clear that items (1) and (2) - the persistence feature mentioned above - would strongly suggest the density wave concept. Indeed, based on these features, we postulated the QSSS hypothesis (hypothesis of quasi-stationary spiral structure), which enabled us to explain a large body of observational data. It also raised a number of interesting questions on the dynamical mechanisms in stellar systems; e.g., the behaviors of material arms and of density waves, the origin of density waves, the unique- 
ness of the spiral pattern, and the behavior of stars, collectively and individually, near the points of co-rotation and Lindblad resonance*.

We can expect the existence of density waves also to have deep implications on the physical processes in the interstellar medium, and in particular on the formation of new stars. We can also expect the success of the theory, as applied to the Milky Way System, to embolden us to apply the theory to external galaxies. These are indeed fruitful avenues for future research work.

We therefore take the existence of density waves, and in particular, the QSSS hypothesis as our central theme. On the one hand, we examine its implications in our own galaxies and in other galaxies. On the other hand, we look for the mechanism for its origin, and use this as a starting point to examine many interesting behaviors of the stellar system.

One important theme to be kept in mind is COEXISTENCE. The complicated spiral structure of the galaxies indicates the coexistence of material arms and density waves, - and indeed of the possible coexistence of several wave patterns. When conflicting results appear to be suggested by observations, the truth might indeed lie in the co-existence of several patterns. Before taking this 'easy way out', one should of course try to examine each interpretation of the observational data as critically as possible.

There is also coexistence in the problem of origin of spiral structure. From our experience with plasma physics, we learned that there are many types of instabilities. Since a stellar system is basically a plasmoidal system, various types of instability can also occur in the problem of the galactic disk. The instability mechanism suggested in Section 3 is therefore only one of several possibilities. It has the advantage of being 'gross' or 'ponderous' instead of being 'delicate'. Such 'gross' instabilities, whenever they exist, usually occur prominently (as our experience in problems of hydrodynamic instabilities tells us). Another 'gross' mechanism for the origin of spiral structure is the existence of a short bar at the center, whose formation may or may not be related to the mechanism discussed in Section 3.

\section{DISCUSSION OF THE GENERAL FEATURES}

To go beyond items (1) and the first part of (2), we need to work out the detailed dispersion relationship for density waves. A description of the explanation of the second part of item (2), items (3), (4), and (5) may be found in the paper by Lin et al. (1969). We note especially that the ring structure is associated with Lindblad resonance. Items (6), (7), (8), and (9) can be explained in terms of a sudden compression of the gas, as it passes through a density wave, as will be discussed in some detail by W. W. Roberts (this volume, p. 415; see also Roberts, 1969). Clearly, the role of magnetic field (item (10)) is secondary in our picture.

* When the stars meet the density wave at a frequency equal to its epicyclic frequency, there is a resonance behavior. This is referred to as Lindblad resonance (cf. the lecture of Contopoulos, this volume, $p$.303). There is of course also 'resonance' behavior when the stars move with the $w$ ave. This is however called specifically co-rotation to avoid confusion. 
The detailed discussion, as presented at the conference, will not be given here. I wish only to record the following remarks.

1. The existence of the primary dust lane on the inner side of the bright spiral arm is compatible with a trailing spiral pattern travelling at a pattern speed lower than the material speed (cf., Lynds, this volume, p. 26).

2. The separation between the dust lane and the bright spiral arm should be, according to the theory, a measure of the difference between the pattern speed and the material speed (cf., Roberts, this volume, p. 415). It should therefore be larger in the interior part of the galaxy (cf., Morgan, this volume, p. 9) where the angular velocity of the material motion is larger, the angular velocity of the pattern being independent of radial distance.

3. As we move away from the center of the galaxy, formation of new stars and hence the bright spiral pattern should terminate where the material speed and the pattern speed become nearly the same. This comment is very useful for determining the pattern speed in external galaxies.

4. External galaxies should be studied in great detail for confirmation of these ideas. In particular, the ring structure deserves special attention, since extraordinary behavior may be expected near resonance.

5. Secondary dust lanes across a spiral arm ('feathers') or on the outer edge of an arm are probably related to the visibility of the dust and other secondary dynamical behavior in an arm. Dust is probably present throughout an arm. These points deserve further attention, as they will cast light on the mechanisms occurring within a spiral arm.

\section{Spiral Structure of the Galaxy}

\section{A. A BRIEF DESCRIPTION OF THE SPIRAL STRUCTURE}

Having stated my general position, I shall proceed directly to a description of our conclusions. In Figure 1, we show an old picture already published in the Proceedings of the Noordwijk Symposium. It shows a theoretical pattern of our galaxy that is in reasonable agreement with observations. This diagram gives the skeleton of the spiral structure calculated according to the density wave theory, as applied to a basic galactic model given by Schmidt (1965). The pattern is trailing, and rotating around the Galaxy at a pattern speed of $11 \mathrm{~km} \mathrm{~s}^{-1} \mathrm{kpc}^{-1}$. Thus, the material motion is faster than the wave pattern. According to the calculations of W.W. Roberts (which are qualitatively in agreement with the earlier calculations of Fujimoto for other values of the parameters involved), this leads to a sharp rise of the gas density (on the inside of the optical spiral arm) followed by a sudden expansion (towards the outside of the spiral arm). We could infer from this that the dust lane would be expected to occur close to the inner edge of the bright optical arm.

The pattern speed of $11 \mathrm{~km} \mathrm{~s}^{-1} \mathrm{kpc}^{-1}$ is now considered a lower limit; a more accurate value is perhaps $11.5 \mathrm{~km} \mathrm{~s}^{-1} \mathrm{kpc}^{-1}$, if we continue to adopt a model of an infinitesimally thin disk. The detailed calculations were made by Yuan, and will 
be discussed by him in detail. I shall merely mention here that the spiral pattern derived by him from the observational data differs only slightly from the theoretical pattern. It must be recognized that the agreement should not be perfect, since the galactic disk is perhaps not perfectly circular and the actual structure may not be a pure mode in the theory.

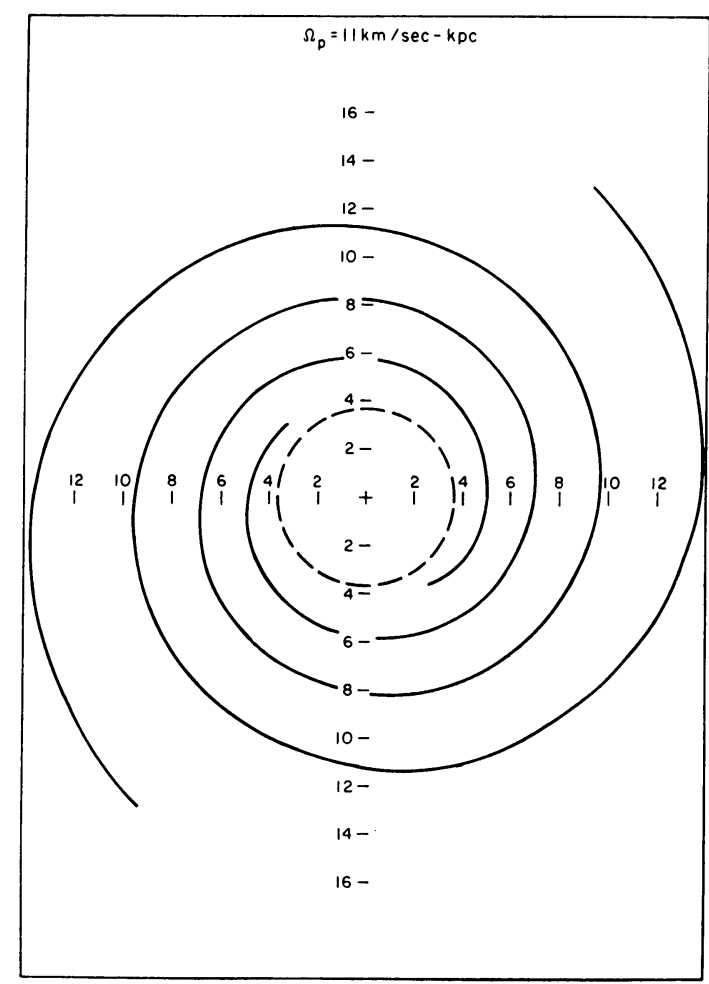

Fig. 1. Spiral pattern for 1965 Schmidt model.

When the thickness of the galactic disk is considered, Shu found that the pattern speed should be approximately $13.5 \mathrm{~km} \mathrm{~s}^{-1} \mathrm{kpc}^{-1}$ in order to reproduce the observed spiral pattern. These calculations were made on the basis of his approximate evaluation of the thickness effect. At this symposium, Vandervoort (p. 341) reported further and more careful investigations of the effect of thickness of the galactic disk. The results agree very well with the earlier results obtained by Shu.

Other parameters that characterise the nature of the primary density wave are also shown in Table I. For a complete discussion of the manner in which these parameters are determined, the reader is referred to the paper by Lin et al. cited above.

The pattern speed we obtained is in sharp contrast with the value of $32 \mathrm{~km} \mathrm{~s}^{-1}$ $\mathrm{kpc}^{-1}$ obtained by Kalnajs. One can reconcile the two different results only if we accept the idea that there may be more than one mode present in the galaxy. The mode 
we obtained can be compared with a number of observational data, such as (a) the distribution of neutral hydrogen and its correlation with the oscillations in the rotation curve, (b) the distribution of young stars, (c) the origin of the local stars, and (d) the vertex deviation of the velocity distribution of the local stars. The discussion of items (a), (b) and (c) may be found in the paper by Lin et al.; item (d) will be discussed in a forthcoming paper by Strömgren, Lin and Yuan. One principal result is that the velocity distribution of the A stars, as observed by Eggen, can be very well reproduced by theoretical calculations based on the parameters shown in Table I.

\section{TABLE I}

Certain dynamical parameters

1. rms radial velocity predicted for stars in the solar neighborhood

$$
\begin{aligned}
& \leqslant 37 \mathrm{~km} \mathrm{~s}^{-1} \\
& \quad 13.5 \mathrm{~km} \mathrm{~s}^{-1} \mathrm{kpc}^{-1}
\end{aligned}
$$

2. Spiral pattern (primary component), pattern speed

3. In the solar vicinity
(a) arm spacing (between Perseus and Sagittarius arms)
$3.5 \mathrm{kpc}$
(b) amplitude of spiral gravitational field
(c) amplitude of variation of projected mass density
(i) in stars
(ii) in gas
(d) rms turbulent velocity of the gas (adopted)
(e) magnetic field (adopted for dynamical consistency)
$5 \%$ of mean field
$10 \%$ of mean
$5 \%$
$5 \%$
$7 \mathrm{~km} \mathrm{~s}^{-1}$
$5 \mu \mathrm{G}$

\section{B. SOME SPECIAL PROBLEMS}

I wish now to call attention to three special problems which have been raised from time to time. The answers to these questions are not entirely clear, but I shall offer some opinions and comments.

(1) Do stars and gas move together?

(2) What is the nature of the local arms:

(i) the Carina arm, (ii) the Orion arm,

(iii) the Perseus arm, (iv) the Sagittarius arm?

(3) What are the $3 \mathrm{kpc}$ arms?

(1) During this Symposium, Kerr has offered observational evidence that the $\mathrm{H}_{\mathrm{I}}$ and $\mathrm{HII}$ regions move at approximately the same velocity, within a scatter of about $10 \mathrm{~km} \mathrm{~s}^{-1}$. According to the gravitational picture, the stars and gas should move together, in the mean, provided that they are located at the same point in space, and that their velocity dispersions (turbulence in one case, peculiar velocity of stars in the other) exert a similar influence. Neither condition is exactly satisfied when one observes in a given direction. Thus, a small amount of deviation does not give evidence to the existence of other types of forces in action.

(2) The local arms are not necessarily all of the same nature. This is a godsent 
opportunity for us to understand the various physical processes, through theoretical considerations and the complementary use of a variety of observations. The Perseus and the Sagittarius arms fit into our large scale structure. The Orion arm is presumably a material arm (possibly formed out of material separated from the Sagittarius arm about $80-100 \times 10^{6} \mathrm{yr}$ ago). The Carina arm could be composed of young objects migrated out of the Sagittarius arm. It may also be a part of a trailing pattern travelling at about $15 \mathrm{~km} \mathrm{~s}^{-1} \mathrm{kpc}^{-1}$. There is also the possibility that it might be a part of a trailing pattern, travelling at a speed higher than the local circular speed. Such an arm would have its dust lane on the outside of its bright objects.

(3) The ' $3-\mathrm{kpc}$ arm' is a deep mystery, as discussed in some detail in the forthcoming paper by W. W. Roberts (1969). One more suggestion might be made here. If indeed the mechanism suggested in Section 3 is correct, then the ' $3-\mathrm{kpc}$ arm' is a part of a reflected leading wave, of an evanescent type (i.e., one whose amplitude decreases exponentially in space as it leaves the ring of reflection). Such a wave carries no energy flux, the reflected energy being carried outwards by the long waves described in Section 3. This picture is necessarily tentative. A careful study of the reflection mechanism is therefore an urgent matter to prove or disprove the conjecture.

\section{On the Origin and the Maintenance of Galactic Spirals*}

\section{A. OVERVIEW}

Having provided a possible mechanism for a grand design over the whole galactic disk, we must now investigate the origin and the long term maintenance of the density waves that underlie the galactic spiral structure. In his survey lecture at the beginning of this Symposium, Oort raised this question. The consideration of the propagation of a group of density waves (Toomre, 1969) brings the problem even into sharper focus. In the following discussions, we suggest a mechanism for the initiation of trailing spiral waves and for the maintenance of a two-armed spiral pattern in a quasistationary manner. In our picture, trailing spiral arms, co-rotating with the general mass motion, are produced by stretching of irregularities at the outer parts of a galaxy through differential rotation. Owing to resonance, the two-armed structure will prevail as the disturbances propagate inwards as a group of waves, which extracts energy from the basic rotation of the galaxy. The pattern speed expected from this mechanism in our Galaxy is thus around $11-13 \mathrm{~km} \mathrm{~s}^{-1} \mathrm{kpc}^{-1}$, consistent with that determined from observational data (Lin et al., 1969).

The reflection of the waves from the central region then stabilizes the wave pattern into a quasi-stationary form by transmitting the signal, via long-range forces, back to the outer regions where the waves originated. Thus, there is necessarily the coexistence of a very loose spiral structure and a tight spiral structure. Population I objects stand out sharply in the tight pattern while stars with large dispersive motion would primarily participate in the very loose pattern.

\footnotetext{
* The author is grateful to F. Shu and A. Toomre for several helpful discussions.
} 


\section{B. A STRETCH OF A TRAILING SPIRAL ARM PRODUCED BY DIFFERENTIAL ROTATION}

Since the galactic disk is in a state of strong differential rotation, any structural irregularity is likely to be stretched into a part of a trailing spiral arm, moving approximately at the local circular velocity, and inclined to the circular direction at various and varying angles. This material arm exerts its own induced effect and it may be expected that it eventually becomes a roughly self-sustained entity, somewhat like the self-sustained density waves (Lin and Shu, 1964, 1966, 1967) with inherent frequency $v=0$ (co-rotating waves). The process of such an evolution may be followed by the theoretical studies* of Goldreich and Lynden-Bell (1965) and of Julian and Toomre (1966).

Where would such structural irregularities most likely occur? The answer seems to be: in the outer regions of the galaxy. There is observational evidence for this statement: connecting links between major spiral arms are frequently observed in the outer parts of many galaxies. In our opinion, the Orion arm is such an interarm branch (Lin et al., 1969). There is also a theoretical basis for this conjecture. The Jeans instability of the galactic disk (Toomre, 1964) tends to produce irregularities unless it is stabilized by the peculiar velocities of the stars and the turbulent motion of gas. Since the stellar content is smaller in the outer parts, and the turbulent motion of the gas may be dissipated, these outer parts of a galaxy are likely to be gravitationally unstable. Structural irregularities develop and are stretched out into short spiral arms, co-rotating with the prevailing local circular motion. Such a disturbance $q(\varpi, \theta, t)$, at a distance $\varpi=\varpi_{0}$ from the galactic center may be represented by a formula of the general form

$$
q=F\left(t / \tau_{0}, \quad \theta-\Omega_{0} t\right),
$$

where $\Omega_{0}$ is the angular velocity at $\varpi_{0}$, and $\tau_{0}$ is a time scale much longer than $2 \pi / \Omega_{0}$. We shall henceforth neglect the slow dependence on time indicated by the argument $t / \tau_{0}$.

\section{RESONANCE}

The disturbance in the form of a piece of a co-rotating spiral arm would naturally exert its influence on other parts of the galaxy, and could induce density waves. But in general its effect would be expected to be limited, unless there is some form of resonance. A 'near-resonance' mechanism** becomes apparent when we consider the individual harmonic components of (1) and concentrate on the component with $2 \theta$ dependence.

* The analysis made by these authors becomes inconclusive for application to waves on a finite disk when the angle of inclination becomes small; for the radial distance of the waves from the galactic center was effectively taken to be infinite. We therefore do not believe that the waves would eventually be wiped out by Landau damping, as indicated by Julian and Toomre.

** As pointed out by G. Contopoulos, the 'near resonance' considered here is, strictly speaking, still quite far away from the resonance condition defined by Equation (5). The term as used here merely indicates that the waves can propagate over a substantial part of the disk where $\left|2\left(\Omega-\Omega_{0}\right)\right|<\kappa$, which defines the "principal part of the spiral pattern" (Lin and Shu, 1966). 
Let us write

$$
q=\sum_{m=0}^{\infty} q_{m} e^{i m\left(\theta-\Omega_{0} t\right)}
$$

and consider the effect of the individual components. The stars at a galacto-concentric distance $\varpi$ will feel a gravitational field at an angular frequency

$$
f=m\left[\Omega(\varpi)-\Omega_{0}\right]
$$

If this angular frequency $f$ is nearly equal to the epicyclic frequency $\kappa(\varpi)$, then there is near-resonance, and we may expect the disturbance (2) to have a large effect. The condition of resonance is

$$
\kappa(\varpi)=m\left(\Omega(\varpi)-\Omega_{0}\right)
$$

or

$$
\Omega_{0}=\Omega(\varpi)-\kappa(\varpi) / m .
$$

Clearly, this can be satisfied only for one particular value of $\varpi$. However, near-resonance may be expected for a wide range of values of $\varpi$ if the right-hand side of (5) is nearly constant. In the outer parts of a galaxy, the variations in $\Omega(\varpi)$ and $\kappa(\varpi)$ are small. Thus, the required condition may be nearly satisfied for a substantial range of the radial distance $\varpi$ for a few values of $m$. In the inner parts of a galaxy, the required condition can be satisfied only for $m=2$. (In the case of uniform rotation, $\Omega-\kappa / 2$ is exactly equal to zero.) This is a fact noted by B. Lindblad, and it has played an important role in the theory of density waves.

It is thus possible to conclude that the component of (2) with $m=2$ could produce a substantial effect in the whole galaxy, provided it occurs at such a galacto-centric distance that $\Omega_{0}$ is nearly equal to the nearly constant value of $\Omega(\varpi)-\kappa(\varpi) / 2$. In our galaxy, this means that

$$
\Omega_{0}=11-13 \mathrm{~km} \mathrm{~s}^{-1} \mathrm{kpc}^{-1} \text {. }
$$

and that the disturbances should originate around $\varpi_{0}=15 \mathrm{kpc}$. This is indeed a region where the stars are less abundant, and hence the system is gravitationally unstable.

\section{PROPAGation OF THE Disturbance}

There is therefore the possibility of a multiple-armed structure at the outer reaches of a galaxy, but all prominent spiral structures are expected to be two-armed. The spiral structure consists of a group of trailing waves with phase velocity (in angle)

$$
\Omega_{p}=11-13 \mathrm{~km} \mathrm{~s}^{-1} \mathrm{kpc}^{-1} \text {. }
$$

Let us now consider how these waves would behave as they propagate in the radial direction.

There are two possible groups of trailing waves (Toomre, 1969): in the range $\Omega>\Omega_{p}$, they are (i) the short waves with a negative group velocity, and (ii) the long 
waves with a positive group velocity. Clearly, only the short trailing waves will prevail*, if the disturbances are initiated in the manner described above.

It is of course too much to expect that these short trailing waves would naturally produce a quasi-stationary spiral pattern. In the first place, the energy supplied by the disturbances, randomly created at the outer regions, must be limited. Furthermore, it will disperse during propagation. Secondly, the 'slow' dependence on the time scale $\tau_{0}$ is bound to show up fairly soon, and a well-organized pattern is therefore unlikely to emerge. How can these two difficulties be met?

The first difficulty, we shall see, will be resolved by the feeding of energy from the differentially rotating galactic disk. The second difficulty will be resolved by a reflection of the wave from the central regions of the galaxy, either at a resonance ring or at the galactic center, where a more-or-less bar-shaped structure will be formed.

\section{E. ENERGY FEEDING DURING PROPAGATION}

It is easy to imagine that a differentially rotating disk can exchange energy with waves propagating through it, but one must determine whether the waves gain or lose energy. Also, one must consider the associated re-adjustment of the distribution of the rotation of the galaxy; the time scale of this adjustment should be very long. This latter point is easy to settle. A rough estimate of this time scale is $\varepsilon^{-2}$ times a typical period of revolution, where $\varepsilon$ is the fractional variation of the various quantities in the disturbance. If we take $\varepsilon$ to be of the order of $10 \%$, the time scale for this perennial adjustment would be of the order $10^{10} \mathrm{yr}$.

The feeding of energy can be calculated quantitatively. It is known that, in many cases of wave propagation, there is a principle of conservation of density of action (Whitham, 1965; Bretherton and Garrett, 1967). In the case of density waves under consideration, this principle has also been found to be true by Toomre (1969)** based on results obtained by Shu (1968).

Briefly, this principle may be stated as follows. If the energy density of the wave is denoted by $\mathscr{E}$, we define the density of action by

$$
\mathscr{A}=\mathscr{E} \mid f \text {, }
$$

where $f$ is the angular frequency seen by an observer co-moving with the general stream. It then follows that

$$
\partial \mathscr{A}+\nabla \cdot\left(\mathscr{A} \mathbf{c}_{g}\right)=0
$$

where $\mathbf{c}_{\boldsymbol{g}}$ is the group velocity.

* The waves outside the co-rotation point would also be short trailing waves, since they propagate outwards where $\Omega<\Omega_{p}$. Note that $\Omega(\varpi)$ decreases with increasing values of $\boldsymbol{\varpi}$.

** Toomre's calculations were made for axisymmetric modes, for which the effect of change in frequency is not evident. The extension to non-axisymmetric modes, although natural to anticipate, does involve rather non-trivial calculations. This was completed by Shu. During this Symposium, Kalnajs pointed out that the energy density defined here is that associated with the rotating coordinate system. This does not alter the main line of reasoning here, especially that part related to the vanishing of the group velocity near resonance. 
Following the motion of the group of waves, we then have, by (9),

$$
\left(\begin{array}{c}
\mathrm{d} \mathscr{A} \\
\mathrm{d} t
\end{array}\right)_{\mathbf{c}_{g}}=\frac{\partial \mathscr{A}}{\partial t}+\left(\mathbf{c}_{g} \cdot \nabla\right) \mathscr{A}=\mathscr{A}\left(\nabla \cdot \mathbf{c}_{g}\right),
$$

which may also be written as

$$
\left\{\frac{\partial}{\partial t}+\left(\mathbf{c}_{g} \cdot \nabla\right)\right\} \log \mathscr{A}=-\nabla \cdot \mathbf{c}_{g},
$$

or

$$
\left\{\frac{\partial}{\partial t}+\left(\mathbf{c}_{g} \cdot \nabla\right)\right\} \log \mathscr{E}=\left\{\frac{\partial}{\partial t}+\left(\mathbf{c}_{g} \cdot \nabla\right)\right\} \log f-\nabla \cdot \mathbf{c}_{g} .
$$

If the right-hand side of the above equation is positive, we have an amplification of the wave group during propagation. If it is negative, we have an attenuation. In the present case, we shall find that the right-hand side is indeed positive, and we have consequently an amplification of the disturbance energy as the wave propagates inwards, when the waves are stationary $(\partial() / \partial t=0)$. This is very easy to verify since

$$
c_{g}<0, \text { and } \frac{\mathrm{d} \Omega}{\mathrm{d} \varpi}<0 .
$$

The form (9) yields a more vivid description of the mechanism. In the stationary case, we have

$$
\varpi c_{g} \mathscr{A}=\text { constant } .
$$

Near the point of origin, the angular frequency $f$ (Equation (3)) is very small. Consequently (Equation (8)), it requires only a negligible amount of energy to produce a substantial amount of action. As the group of waves propagates inwards, the angular frequency $f$ steadily increases, and the energy density $\mathscr{E}$ must therefore increase proportionally. In addition, there is further enhancement of $\mathscr{E}$ due to the decrease of $\left|c_{g}\right|$ and $m$. Indeed, if there is a point of inner Lindblad resonance, $\left|c_{g}\right| \rightarrow 0$ there*, and the energy density becomes very large. The increase of energy density with decreasing $\varpi$ is of course to be expected from the cylindrical geometry of the galactic system.

We note however that the mechanism will not be operative unless there is a preexisting mechanism for wave propagation. Furthermore, if the propagation mechanism is imperfect, i.e., if energy may be partly lost by dispersion, the effect of this gradient instability may be somewhat obscured. In the present case, we are fortunate to have a condition of near-resonance, which tends to insure a favorable propagation of the waves.

\section{F. REFLECTION OF WAVES NEAR THE CENTRAL REGION}

From the discussions of the last section, there appear two possibilities for the action of the wave group to become infinite, if we insist on using Equation (14). Either we

* Indeed, it vanishes as $(1-|v|)^{3 / 2}$. 
have (i) $\varpi=0$, or we have (ii) $c_{g}=0$. The first condition refers to the galactic center, and the second condition refers to Lindblad resonance. These are the places where the propagation of the group of waves must undergo a change, and it is natural to think of them as places where the waves, having reached a very large (but not infinite) magnitude, are reflected. Let us examine the consequences of this reflection process, beginning with the galactic center.

As the group of waves approaches the galactic center, its greatly increased amplitude will be sufficient to cause the galactic nucleus to be slightly distorted* into a 'short bar'. This 'bar' configuration will be rotating at an angular velocity $\Omega_{p}$, appropriate to the forcing incoming wave. There is thus an associated gravitational field also rotating at the same angular frequency $\Omega_{p}$, propagating outwards. Its effect will be particularly strongly felt by the outer rings of the galaxy where the circular velocity is $\Omega_{p}$; i.e., where the waves originated. Thus, the cycle does complete itself and a stationary state may be maintained.

The completion of the cycle must be done with the right phase in time and right distribution in space. An indication for this possibility may be seen by examining the outward propagation. If we persist in applying the results obtained for the short waves to the relatively long waves, we find that the two branches coincide for the condition of co-rotation $v=0$. Thus, if the energy is carried out by the long trailing waves, it would be forcing the outer parts of the galactic disk with an appropriate 'mode' (i.e., an appropriate distribution in space and variation in time) provided one numerical value characterizing the phase is correctly specified. Since we are dealing with a group of waves whose 'wave length' is quite long, and not really well defined, this is not a critical requirement. At the same time, one cannot expect the re-enforcement to be perfect, but this is again not a critical matter. A partial feedback would be able to sustain a relatively stationary pattern, since there is a substantial gain of energy as the short waves move inwards, but not a corresponding loss of energy as the long waves, with length scale on the order of the galactic radius, propagate outwards.

\section{G. INNER RESONANCE RING}

In cases where a sharp Lindblad resonance exists (e.g. in NGC 5364 or the Milky Way), the waves cannot penetrate into the center, but would be reflected already at the resonance ring. The violent response of the stars suggests that they are likely to group themselves into certain orbits, collectively showing an oval structure (cf., Contopoulos, this volume, p. 303), which takes the place of the bar in the above described reflection mechanism; but the general points made above still hold. The precise mechanism of reflection is obviously a very important and very interesting process deserving much future attention.

There is the distinct possibility that the waves would be absorbed at the inner resonance ring, and that there is no reflection of the group of waves. If this were to be found to be the case, there would be difficulty in accounting for the regular orga-

* In a pure disk model, the 'short bar' thus produced at the center would presumably be represented by a singularity. See Lin and Shu (1964), Rehm (1965), or as quoted by Lin and Shu (1967). 
nization of a grand design. It would then be more likely that we would see a rather chaotic superposition of two-armed waves, unless certain nonlinear effects related to resonant stars (Contopoulos, 1970), would prevail.

\section{H. CONCLUSION}

We have thus arrived at a picture involving the co-existence of a relatively tightly wound pattern of short waves and a rather loosely wound pattern of long waves. Clearly, objects with small peculiar velocities - particularly Population I objects would participate in the tight spiral pattern. On the same length scale, the loosely wound pattern, with at most one winding for the whole galaxy would present itself as a distortion of the whole galaxy. It would be less visible to casual optical and radio observations. It could conceivably be mapped out via very careful analysis of optical and radio data.

All the above discussions are still fairly speculative. It would be extremely desirable to follow through the detailed development of these ideas with more careful mathematical analysis.

\section{Concluding Remarks: A Bird's Eye View of Theoretical Developments}

As mentioned before, we use the QSSS hypothesis as the focal point for our theoretical developments. On the side of basic mechanism, we raise various dynamical issues whose clarification may also help us to understand the behavior of electro-magnetic plasmas. For the study of our own galaxy, and perhaps other galaxies as well, there appear to be a better opportunity for the understanding of the physical processes, including such microscopic behavior as the formation of molecules and dust grains, induced by macroscopic compression via density waves. The effect of the density wave can also influence the cosmic ray particles via the galactic magnetic field. Here it is important to keep alive the general concept of density waves, and not to attach it only to a two-armed grand design.

We have also the opportunity to explore the external galaxies, since here certain types of observations can be made more easily*, and we have a larger sample of objects for our study. The relationship between normal spirals and barred spirals is a fascinating subject. It is perhaps sufficient, but not necessary, to have a short barred structure at the center of a normal galaxy, to initiate a two-armed grand design. The coexistence of several origins for spiral structure should be stressed. Simultaneously, there is the possibility of the coexistence of several kinds of spiral structures and their actual revelation as observable features. Indeed, it is the feeling of the present writer that relatively loose spiral patterns tend to be obtained by computational or analytical methods directed at the whole galactic disk. The asymptotic method yields relatively tight spirals when near resonance is approached, but it can also yield fairly loose spirals.

* One must beware of possibilities of misinterpretation, for we can only measure the velocity in the line of sight. Since the material in a galactic disk has two other components of motion besides that in the circular direction, inferences on the small deviations in circular motion must be drawn with care. 


\section{Acknowledgement}

The work reported here has been supported in part by grants from the National Science Foundation and the National Aeronautics and Space Administration.

\section{References}

Bretherton, F. P. and Garrett, C. J. R.: 1967, Proc. Roy. Soc. London A302, 529.

Contopoulos, G.: 1970, Astrophys. J., in press.

Goldreich, P. and Lynden-Bell, D.: 1965, Monthly Notices Roy. Astron. Soc. 130, 97, 125.

Julian, W. H. and Toomre, A. 1966, Astrophys. J. 146, 810.

Lin, C. C.: 1967, in Relativity Theory and Astrophysics, 2: Galactic Structure, Amer. Math. Soc., Providence, p. 66.

Lin, C. C. and Shu, F. H.: 1964, Astrophys. J. 140, 646.

Lin, C. C. and Shu, F. H.: 1966, Proc. Nat. Acad. Sci. U.S.A. 55, 229.

Lin, C. C. and Shu, F. H.: 1967, IAU-URSI Symposium No. 31, p. 313.

Lin, C. C., Yuan, C., and Shu, F. H.: 1969, Astrophys. J. 155, 721.

Oort, J. H.: 1962, in Interstellar Matter in Galaxies (ed. by L. Woltjer), Benjamin, New York, p. 234.

Roberts, W. W.: 1969, Astrophys. J. 158, 123.

Schmidt, M.: 1965, Stars and Stellar Systems 5, 513.

Shu, F. H.: 1968, Ph.D. Thesis, Harvard University.

Toomre, A.: 1964, Astrophys. J. 139, 1217.

Toomre, A.: 1969, Astrophys. J. 158, 899.

Whitham, G. B.: 1965, J. Fluid Mech. 22, 273.

Yuan, C.: 1969, Astrophys. J. 158, 871, 889. 